\title{
Design of Multiple MMSE Subequalizers for Faster-Than-Nyquist-Rate Transmission
}

\author{
Jae-Hyok Lee and Yong-Hwan Lee, Member, IEEE
}

\begin{abstract}
We consider an equalization problem when the transmitted symbol rate is higher than the available channel bandwidth. This situation can happen in the uplink of the voiceband pulse-code modulation (PCM) modems whose transmit signal bandwidth is larger than the available channel bandwidth. Although the use of a minimum mean-squared error (MMSE) pre-equalizer is considered in the International Telecommunications Union (ITU)-T V.92 Recommendation, it may not provide an acceptable performance unless the channel condition is mild. As another approach to this problem, we consider the use of a bank of subequalizers, each of which compensates the part of the channel distortion, enabling the PCM-mode transmission over the channel where the V.92 scheme may fail. In this paper, a multiple subequalizer scheme is optimally designed in the MMSE sense, and its performance is compared with the MMSE pre-equalizer of V.92 in terms of the bit-rate-normalized signal-to-noise ratio.
\end{abstract}

Index Terms-Equalizer, faster-than-Nyquist signaling, minimum mean-squared error (MMSE).

\section{INTRODUCTION}

$\mathbf{T}$ HE pulse-code modulation (PCM) modem of the International Telecommunications Union (ITU)-T V.90 recommendation [1] assumes that one end of the modem access has digital connection to the phone network, which is already used by internet service providers (ISPs) and corporations for remote access. The data bits sent by the ISP modem are converted into appropriate PCM symbols for digital-to-analog (D/A) conversion in the local central office (CO) at a rate of $8 \mathrm{kHz}$ [2], resulting in a maximum data throughput of $64 \mathrm{~kb} / \mathrm{s}$. However, due to the use of the least significant bit (LSB) for other signaling purposes, the maximum transmission rate is limited to $56 \mathrm{~kb} / \mathrm{s}$ in the V.90-mode modem. The PCM symbols from the codec, which are valid channel-symbol alphabets, are then sampled and properly processed by the user modem to recover the information. The main idea behind this technique is to avoid the effect of quantization distortion of the codec by using the quantization levels of digital-to analog converter (DAC) as the channel-symbol alphabets.

While the PCM mode is employed for the downlink connection in the V.90 modem, it cannot be employed for the uplink connection, mainly because of the bandwidth problem. The local loop channel has a spectral null at direct current (dc) due

Paper approved by E. Ayanoglu, the Editor for Communication Theory and Coding Applications of the IEEE Communications Society. Manuscript received August 4, 2002; revised March 9, 2003.

J.-H. Lee is with the Telecommunications Systems Division, Telecommunication Network Business, Samsung Electric Company, Ltd., Suwon City, Gyeonggi-Do 442-600, Korea (e-mail: hyok.lee@samsung.com).

Y.-H. Lee is with the School of Electrical Engineering and Computer Science, Seoul National University, Seoul 151-742, Korea (e-mail: ylee@snu.ac.kr).

Digital Object Identifier 10.1109/TCOMM.2004.833009 to the use of isolation transformers, and the transmit signal from the user modem is strictly bandlimited by low-pass filters in the $\mathrm{CO}$ [3]. The effective channel bandwidth is less than $3500 \mathrm{~Hz}$, while the transmission of the PCM signal requires a bandwidth of at least $4 \mathrm{kHz}$. The PCM-mode transmission in the downlink of V.90 employs a dc-free coding scheme combined with the use of equalization in the receiver. However, it is not possible to directly employ the PCM mode in the uplink of V.90, since the analog-to-digital (A/D) converted samples at the local CO must have a value corresponding to a valid PCM quantization level. This problem was resolved in the ITU-T V.92 Recommendation [4], where two infinite-impulse response (IIR) filters are used in the transmitter to pre-equalize the channel distortion over the 4 $\mathrm{kHz}$ channel bandwidth, enabling the PCM mode transmission in the uplink. The use of the V.92 mode modem can provide a maximum transmission rate of $48 \mathrm{~kb} / \mathrm{s}$ in the uplink.

The pre-equalization scheme of the V.92 modem uses a general MMSE equalization technique that can precompensate the channel distortion of up to $4-\mathrm{kHz}$ bandwidth of the transmit PCM signal. An IIR-type structure is employed to equalize sharp dc spectral null, which can be possible with the use of a finite-impulse response (FIR) filter with an unacceptably large tap length. The maximum tap length of the IIR filter is limited to 320 taps by the V.92 Recommendation. However, it may not be possible to compensate the channel distortion enough for transmission of the PCM signal under certain channel conditions. In this case, the V.92 modem switches back to the V.34 quadrature amplitude modulation (QAM) signaling mode [5] that can provide a maximum transmission rate of $33.6 \mathrm{~kb} / \mathrm{s}$.

As an alternative to the pre-equalization method, the use of a multiple subequalizer structure was proposed in [6]. Instead of using the channel bandwidth of up to $4 \mathrm{kHz}$, as in the V.92 scheme, this equalizer scheme considers the overall system as a faster-than-Nyquist signaling case [7], [8], corresponding to the case when the transmit signal bandwidth exceeds the available channel bandwidth. It is well known that the channel bandwidth $W$ should be larger than or equal to $f_{b} / 2$ to transmit the data at a symbol rate of $f_{b}$ [9]. However, when $f_{b}>2 W$, it is still possible to transmit the user data by making the actual transmission rate less than $2 W$ symbols/s [10]. A simple way to generate such a signal is to permit only $M$ symbols out of $N$ consecutive symbols to be independently chosen, while the remaining $(N-M)$ symbols are determined so as to satisfy the bandwidth restriction. In an ideal bandlimited channel, such signals can be generated by using $M$ different pulse signals for the $M$ user data symbols, while no user data is transmitted during the rest of the $(N-M)$ symbols time interval [7], [8]. However, this method may not be applicable to an arbitrary-shaped channel, since the pulse signals should be redesigned according to the 


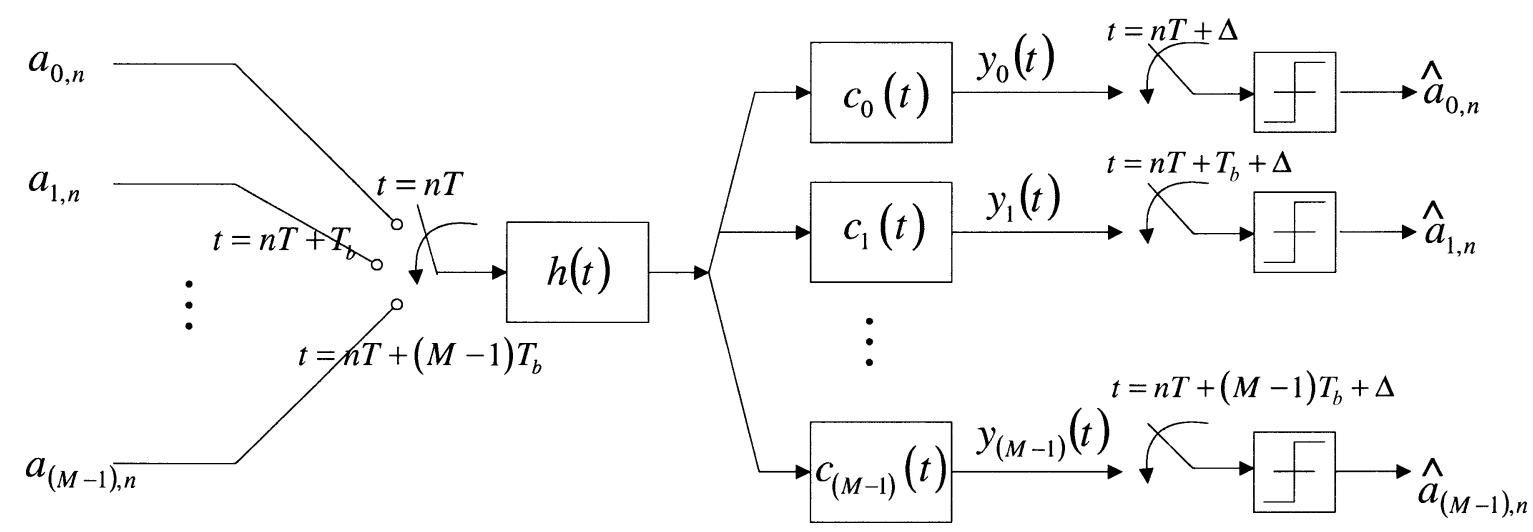

Fig. 1. Equalizer scheme for faster-than-Nyquist signaling.

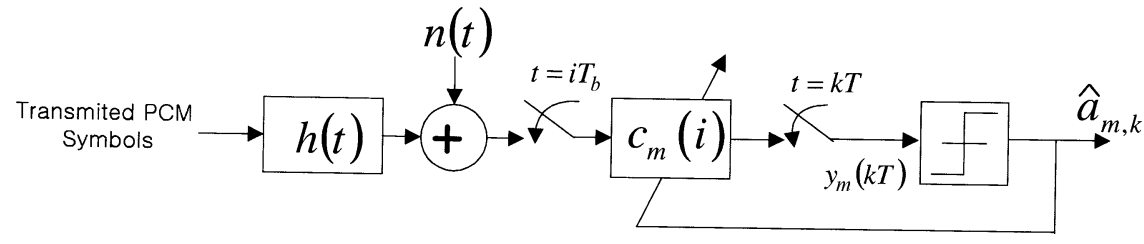

Fig. 2. Equivalent system model for the $m$ th subequalizer with $T=N T_{b}$.

channel shape. This problem can be solved by using $N$ transmit symbol sequences comprised of the $M$ user data and $(N-M)$ zeros with the use of multiple equalizers in the receiver, where $M$ and $N(\geq M)$ are integers such that $M / N \leq 2 W / f_{b}$ [6], [8], [11]. This scheme makes the overall response of the equalizer and channel have the same effect as the signal generated for an ideal bandlimited channel in [7] and [8].

We consider a transmission scheme that employs multiple subequalizers and special formats for the transmit sequence [6]. Although this transmission scheme does not comply with the V.92 Recommendation, it has some advantages over the V.92 pre-equalization scheme. When the channel condition is not mild, the V.92 mode may fail to work, but the proposed scheme works well. However, the maximum transmission rate of the proposed scheme can be lower than that of the V.92 scheme, because the proposed scheme does not use the full 4-kHz bandwidth for transmission of the user information, unlike the V.92 mode. The uniqueness of such a multiple subequalizer scheme was shown using a generalized zero-forcing (ZF) criterion, while a least mean-square (LMS)-type adaptation scheme was applied to the implementation [6]. However, no analytic result has been reported for the performance and properties of the proposed equalizer scheme in the MMSE sense. In this paper, the optimum MMSE coefficient of the multiple subequalizer is analytically designed, and the performance is compared with that of a general MMSE pre-equalizer in the V.92 mode by computer simulation.

Section II describes the transceiver model for the proposed multiple subequalizer scheme. The optimum MMSE coefficient of the multiple subequalizer is analytically derived in Section III. The performance of the proposed equalizer scheme is compared with that of the V.92 pre-equalizer scheme in Section IV. Finally, conclusions are summarized in Section V.

\section{SYSTEM MODEL}

For ease of description, consider the PCM mode transmission in a V.92-class modem [4]. Fig. 1 depicts the structure of the multiple subequalizers proposed in [6], where $h(t)$ represents the impulse response of the channel, and $c_{m}(t), m=0,1, \ldots,(M-$ 1 ) denotes the impulse response of the $m$ th subequalizer, $\Delta$ is the sampling-time offset in the receiver, $T_{b}$ is the PCM symbol rate equal to $1 / 8000 \mathrm{~s}$, and $T=N T_{b}$. To accommodate the available channel bandwidth, every $N$ transmit PCM symbols is comprised of the $M$ user data and $(N-M)$ consecutive zeros as [6]

$$
\begin{array}{r}
\cdots a_{0, n} a_{1, n} \cdots a_{M-1, n} 0 \cdots 0 a_{0, n+1} a_{1, n+1} \\
\cdots a_{M-1, n+1} 0 \cdots 0 \cdots
\end{array}
$$

where $a_{m, n}$ denotes the user data at time $t=(n N+m) T_{b}$.

In the receiver, each subequalizer is used to detect every $N$ th transmit symbols. That is, the $m$ th subequalizer recovers only the $m$ th symbol $a_{m, n}$ in a block of the $N$ received symbols. As shown in Fig. 1, although the received symbols are input to all the subequalizers, each subequalizer generates the output and updates its coefficients only once per every $N$ received PCM symbols. For example, when $N=8$, the subequalizer $c_{0} t$ ) would be adapted based on the difference between its $N T_{b}$ sampled output and the desired sequence

$$
\cdots x a_{0, n} x x x x x x x a_{0, n+1} x x x x x x x a_{0, n+2} x \cdots
$$

where " $x$ " represents the "don't care" symbol. As shown in Fig. 2, the coefficient $c_{m}(t)$ of the $m$ th subequalizer is updated once for each $N$-symbol time, using the error signal corresponding to the symbols $\cdots a_{m, n}, a_{m, n+1}, a_{m, n+2} \cdots$.

Although the equalization scheme in Fig. 1 can be employed in the receiver for the downlink PCM mode communications, it may be desirable to be placed in the transmitter for the uplink 


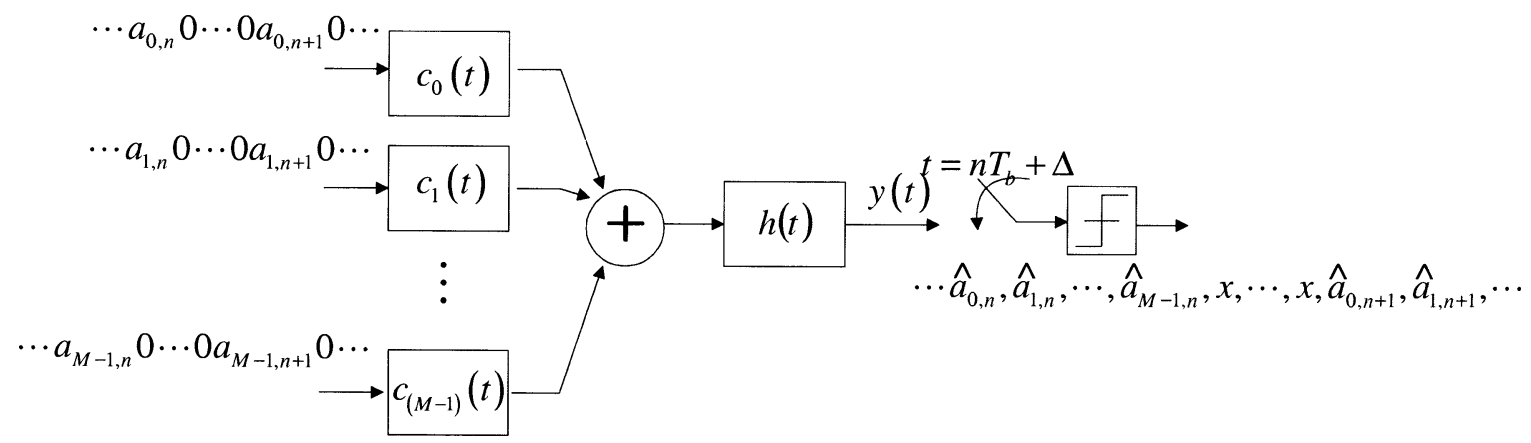

Fig. 3. Pre-equalization scheme for the uplink.

PCM mode connection, so that the output of the A/D converter in the $\mathrm{CO}$ can be free from intersymbol interference (ISI). The corresponding equalizer scheme in the transmitter for the uplink is depicted in Fig. 3. Because the channel follows the equalizer in the uplink, it is not possible to get the error signal for updating the equalizer coefficient. Thus, the adaptation of the equalizer in the uplink should be different from that in the downlink. As in the precoder case [12], the coefficient of the pre-equalizer is trained in the receiver during the initialization process, and then it is sent to the transmitter to set up the coefficient of the pre-equalizer. This coefficient is used during the normal data transmission.

As an example, the pre-equalizer $c_{0}(t)$ can be trained using the transmit signal generated by a pseudorandom sequence

$$
\cdots x a_{0, n} 00000 x x a_{0, n+1} 00000 x x a_{0, n+2} 0 \cdots \text {. }
$$

It is necessary to make the equalizer output at the time of symbol "zero" such that the other transmitters (i.e., other pre-equalizers) can send out their own data without any ISI. At the time of don't-care symbol " $x$," no coefficient is adjusted, and each pre-equalizer uses these " $x$ " symbol times for pulse shaping of the transmit symbol whose rate is faster than Nyquist signaling.

In the next section, we analytically design the optimum equalizer for both the downlink and the uplink in the MMSE sense. Note that the MMSE coefficients can be used for fast initialization of the equalizer.

\section{MMSE EQUALIZATION}

The uniqueness of the proposed equalizer was shown by using a generalized ZF criterion [6]. It is well known that the performance of the MMSE equalizer is better than that of the ZF equalizer, which suffers from the noise-enhancement problem. We consider the MMSE solution of the subequalizer scheme and compare the performance with that of the MMSE equalizer in the V.92 mode.

\section{A. Multiple Subequalizer in the Downlink}

Consider the equalizer scheme in the downlink, as depicted in Fig. 1. An equivalent receiver model of the $m$ th subequalizer $c_{m}(t)$ in an additive white Gaussian noise (AWGN) channel is depicted in Fig. 2, where the channel $h(t)$ includes the transmit and receive filters, as well as the local loop. Define the overall impulse response $w_{m}(t)$ by

$$
w_{m}(t) \equiv \int h(t-\tau) c_{m}(\tau) d \tau \text {. }
$$

Since the transmit sequences are sent in the form of

$$
\begin{aligned}
& \cdots a_{0, n} a_{1, n} \cdots a_{M-1, n} 0 \cdots 0 a_{0, n+1} a_{1, n+1} \\
& \cdots a_{M-1, n+1} 0 \cdots 0 \cdots
\end{aligned}
$$

the output $y_{m}(t)$ of the $m$ th subequalizer can be expressed as

$$
\begin{array}{r}
y_{m}(t)=\sum_{n=-\infty}^{\infty} \sum_{\ell=0}^{M-1} a_{\ell, n} w_{m}\left(t-n T+\ell T_{b}\right)+\nu_{m}(t), \\
m=0,1,2, \cdots, M-1
\end{array}
$$

where $\nu_{m}(t)$ is the noise term of the $m$ th subequalizer output, defined by

$$
\nu_{m}(t) \equiv \int n(t-\tau) c_{m}(\tau) d \tau
$$

Here, $n(t)$ is zero-mean AWGN with a two-sided power spectral density of $N_{0} / 2$.

Define the mean-squared error (MSE) of the $m$ th subequalizer output at time $t=k T$ as

$$
\varepsilon_{m}=E\left\{\left[y_{m}(k T)-a_{m, k}\right]^{2}\right\}
$$

where $E\{\cdot\}$ denotes the expectation process. Since $\left\{a_{m, n}\right\}$ can be assumed statistically independent and identically distributed (i.i.d.) with the same power, (4) can be rewritten as [13]

$$
\begin{aligned}
\frac{\varepsilon_{m}}{P_{a}}=\iint\left[\sum_{n=-\infty}^{\infty} \sum_{\ell=0}^{M-1} h\left(n T-\ell T_{b}-t\right) h\left(n T-\ell T_{b}-\tau\right)\right. \\
\left.+\sigma^{2} \delta(t-\tau)\right] c_{m}(t) c_{m}(\tau) d t d \tau \\
-2 \int h\left(m T_{b}-t\right) c_{m}(t) d t+1
\end{aligned}
$$

where $P_{a}$ is the power of $\left\{a_{m, n}\right\}$ and $\sigma^{2} \equiv N_{0} / P_{a}$. The minimum MSE is found by making the first variation of (5) with respect to $c_{m}(t)$ equal to zero. This results in

$$
\begin{array}{r}
\int\left[\sum_{n=-\infty}^{\infty} \sum_{\ell=0}^{M-1} h\left(n T-\ell T_{b}-t\right) h\left(n T-\ell T_{b}-\tau\right)+\sigma^{2} \delta(t-\tau)\right] \\
\times c_{m}(\tau) d \tau=h\left(m T_{b}-t\right)
\end{array}
$$


It can be easily shown that the coefficients of the $m$ th subequalizer minimizing the $\operatorname{MSE} \varepsilon_{m}$ are given by

$$
\begin{aligned}
c_{m}(t)=\frac{1}{\sigma^{2}} & {\left[h\left(m T_{b}-t\right)-\sum_{n=-\infty}^{\infty} \sum_{\ell=0}^{M-1} w_{m}\left(n T-\ell T_{b}\right)\right.} \\
& \left.\quad \times h\left(n T-\ell T_{b}-t\right)\right] \\
= & \sum_{i=-\infty}^{\infty} \alpha_{m}(i) s(i) h\left(i T_{b}-t\right)
\end{aligned}
$$

where

$$
\begin{aligned}
\alpha_{m}(i) & = \begin{cases}\frac{\left[1-w_{m}\left(i T_{b}\right)\right]}{\sigma^{2}}, & i=m, m=0,1, \cdots, M-1 \\
0, & ((i))_{N}=1,2, \cdots, N-M \\
-\frac{w_{m}\left(i T_{b}\right)}{\sigma^{2}}, & \text { otherwise }\end{cases} \\
s(i) & = \begin{cases}0, & \text { for }((i))_{N}=1,2, \cdots, N-M \\
1, & \text { otherwise }\end{cases}
\end{aligned}
$$

Here $((\cdot))_{N}$ denotes the modulo- $N$ operation. Thus, $c_{m}(t)$ is determined by a weighted sum of the matched-filter output with $(N-M)$ zeros in every $N$ symbols. The coefficient $c_{m}(i)$ can be obtained by substituting (7) into (5) as a function of the channel correlation and noise characteristics. It can be shown that (refer to Appendix)

$$
\begin{aligned}
\sum_{n=0}^{N-1} R_{n}(f)\left[P_{n}(f) \otimes\right. & \left.A_{m}(f)\right]+ \\
& \sigma^{2} P_{0}(f) \otimes A_{m}(f)=e^{-j 2 \pi f m T_{b}}
\end{aligned}
$$

where $\otimes$ denotes the convolution process and

$$
\begin{aligned}
A_{m}(f)= & \sum_{i=-\infty}^{\infty} \alpha_{m}(i) e^{-j 2 \pi f i T_{b}} \\
& \text { for } m=0,1, \ldots, M-1 \\
R_{n}(f)= & \sum_{k=-\infty}^{\infty} r_{h}(N k-n) e^{-j 2 \pi f(N k-n) T_{b}} \\
& \text { for } n=0,1, \ldots, N-1 \\
P_{n}(f)= & \frac{1}{N} \sum_{i=-\infty}^{\infty} p_{n}(i) e^{-j 2 \pi f i T_{b}} \\
& \text { for } n=0,1, \ldots, N-1 .
\end{aligned}
$$

Here, $r_{h}(N k-i)$ is the channel correlation function defined by

$$
\begin{aligned}
& r_{h}(N k-i) \equiv \int h\left(N k T_{b}-\tau\right) h\left(i T_{b}-\tau\right) d \tau \\
& \qquad \text { for } n=0,1, \ldots, N-1
\end{aligned}
$$

and $p_{n}(i)$ is the impulse train defined by

$$
p_{n}(i) \equiv s(i) s(i-n) \text { for } n=0,1, \ldots, N-1 .
$$

Note that $A_{m}(f)$ cannot be expressed in a closed form, due to the involved convolution process. However, note that discrete samples of $A_{m}(f)$ are required to calculate the coefficient $\alpha_{m}(i)$ in digital implementation.

In general, the span of the impulse response of the local loop in the telephone network can be longer than $128 T_{b}$. However, in practice, the use of 128 sample points is enough to represent the channel impulse response without any substantial performance degradation. Thus, it might be sufficient to use 256 autocorrelation samples of the channel impulse response. Letting $L$ be the number of discrete sample points, (10) can be expressed as

$$
\begin{array}{r}
\sum_{n=0}^{N-1} R_{n}(k)\left[P_{n}(k) \otimes A_{m}(k)\right]+\sigma^{2} P_{0}(k) \otimes A_{m}(k)=e^{-\frac{j 2 \pi f k m}{L}}, \\
k=0,1, \ldots, L-1
\end{array}
$$

where $R_{n}(k), A_{m}(k), P_{n}(k)$, and $e^{-j 2 \pi f k m / L}$ are obtained by sampling $R_{n}(f), A_{m}(f), P_{n}(f)$, and $e^{-j 2 \pi f m T_{b}}$ at frequency $f=k /\left(L T_{b}\right)$, respectively. Letting

$$
K(i, j)=\sum_{n=0}^{N-1} R_{n}(i) P_{n}(j)+\sigma^{2} P_{0}(j)
$$

(14) can be represented in a matrix form as

$$
\mathbf{K A}_{\mathbf{m}}=\mathbf{e}
$$

where $\mathbf{K}$ is an $L \times L$ matrix whose $(i, j)$ th element is $K(i, i-j)$, $0 \leq i, j \leq L-1$, and $\mathbf{A}_{\mathbf{m}}$ and $\mathbf{e}$ are $L$-dimensional vectors whose $i$ th element is $A_{m}(i)$ and $e^{-j 2 \pi i m / L}, i=0,1,2, \ldots, L-$ 1 , respectively. Therefore, $A_{m}(k)$ can be obtained by

$$
\mathbf{A}_{\mathbf{m}}=\mathbf{K}^{-1} \mathbf{e} \text {. }
$$

The computational burden to solve (17) can be significantly reduced by noting that $A_{m}(k)$ is conjugate symmetric, and $P_{n}(k)$ is nonzero only at $N$ positions among the $L$ discrete frequency bins. Therefore, if $L=\mu N$, where $\mu$ is an even integer, it is possible to break an $(L \times L)$ matrix of (16) into $\mu / 2$ matrices of size $(N \times N)$, where the factor 2 comes from the conjugate symmetry of $A_{m}(k)$. Note that each of these reduced matrices of size $(N \times N)$ has a rank of $M$, since the transmitted sequence has only $M$ independent data symbols among every $N$ symbols. Thus, these linear equations can be solved by setting the $(N-M)$ unknowns to arbitrary constants. The frequency-domain MMSE coefficient of the $m$ th subequalizer can be obtained by using $A_{m}(k)$ of (17) in (7) as

$$
C_{m}(k)=\left[P_{0}(k) \otimes A_{m}(k)\right] H^{*}(k), \quad m=0,1, \ldots, M-1
$$

where the superscript $*$ denotes the complex conjugate. Finally, the coefficient $c_{m}(i)$ is obtained by taking the inverse discrete Fourier transform (DFT) of $C_{m}(k)$.

\section{B. Multiple Subequalizer in the Uplink}

In the uplink, it is required to make the composite impulse response $c_{m}(i) \otimes h(i)$ have a zero magnitude at all the sampling times, except at every $N$ th sample position corresponding to the output of the $m$ th subequalizer and at don't-care sample positions. For example, the transmit data sequence for training the subequalizer $c_{1}(t)$ is

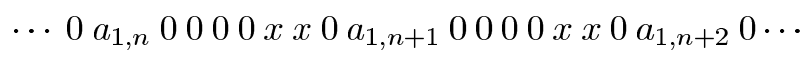

The error signal generated from the subequalizer $c_{1}(t)$ and the above training sequence are used to update the equalizer coefficient. The equalizer coefficient is not updated at the time of 
don't-care sample " $x$. . In this case, the output $y_{m}(t)$ of the $m$ th subequalizer can be expressed as

$$
\begin{aligned}
y_{m}(t)=\sum_{n=-\infty}^{\infty} a_{m, n} w_{m}\left(t-n T+m T_{b}\right)+\nu_{m}(t), & \\
& \text { for } m=0,1,2, \ldots, M-1 .
\end{aligned}
$$

The MSE of the $m$ th subequalizer output at time $t=k T$ is defined as

$\varepsilon_{m}=E\left\{\left[y_{m}(k T)-a_{m, k}\right]^{2}+\sum_{\ell=0, \ell \neq m}^{M-1} y_{m}^{2}\left(k T+(\ell-m) T_{b}\right)\right\}$.

It can be shown that the coefficient $c_{m}(t)$ of the $m$ th subequalizer is expressed as

$$
\begin{aligned}
c_{m}(t)=\frac{1}{M \sigma^{2}}[ & h\left(m T_{b}-t\right)-\sum_{n=-\infty}^{\infty} \sum_{\ell=0}^{M-1} w_{m}\left(n T-\ell T_{b}\right) \\
& \left.\times h\left(n T-\ell T_{b}-t\right)\right] \\
= & \sum_{i=-\infty}^{\infty} \alpha_{m}(i) s(i) h\left(i T_{b}-t\right)
\end{aligned}
$$

where $\alpha_{m}(i)$ is defined as

$$
\alpha_{m}(i)= \begin{cases}\frac{\left[1-w_{m}\left(i T_{b}\right)\right]}{M \sigma^{2}}, & i=m, m=0,1, \ldots, M-1 \\ 0, & ((i))_{N}=1,2, \ldots, N-M \\ -\frac{w_{m}\left(i T_{b}\right)}{M \sigma^{2}}, & \text { otherwise. }\end{cases}
$$

Note that (21) is exactly the same as (7), except for the factor $M$ in the noise power. Letting

$$
K(i, j)=\sum_{n=0}^{N-1} R_{n}(i) P_{n}(j)+M \sigma^{2} P_{0}(j)
$$

the resulting matrix equation can be represented as

$$
\mathbf{K A} \mathbf{A}_{\mathbf{m}}=\mathbf{e}
$$

where $\mathbf{K}$ is an $L \times L$ matrix whose $(i, j)$ th element is $K(i, i-j)$, $0 \leq i, j \leq L-1, \mathbf{A}_{\mathbf{m}}$, and $\mathbf{e}$ are $L$-dimensional vectors whose $i$ th element is $A_{m}(i)$ and $e^{-j 2 \pi i m / L}, i=0,1,2, \ldots, L-1$, respectively. It can be seen from (24) that the MMSE coefficient of the uplink equalizer is exactly the same as the downlink equalizer, except for the factor $M$ in the noise term.

\section{MMSE}

It is of interest to note the relationship between the overall channel $H(f)$ and the MSE at the receiver output. Substituting (6) into (5), we have

$$
\frac{\varepsilon_{m}}{P_{a}}=1-\int h\left(m T_{b}-t\right) c_{m}(t) d t .
$$

Without the loss of generality, it may suffice to consider only the case when $m=0$. If $m=0$, (25) becomes

$$
\frac{\varepsilon_{0}}{P_{a}}=1-\int h(-t) c_{0}(t) d t=1-\int H(f) C_{0}(f) d f .
$$

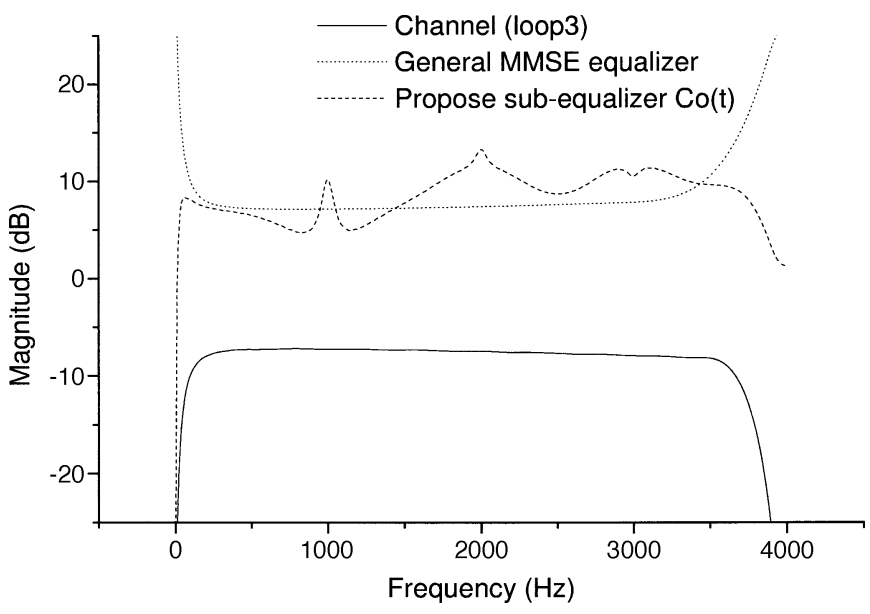

Fig. 4. Frequency response of the subequalizer.

Sampling (26) at $f=k /\left(L T_{b}\right)$ and using (18), we have

$$
\begin{aligned}
\frac{\varepsilon_{0}}{P_{a}} & \approx 1-\sum_{k=0}^{L-1} H(k) C_{0}(k) \\
& =1-\sum_{k=0}^{L-1}|H(k)|^{2}\left[P_{0}(k) \otimes\left(\mathbf{K}^{-\mathbf{1}} \cdot \mathbf{1}\right)\right]
\end{aligned}
$$

where 1 is a unit vector of size $(L \times 1)$. Note that the MSE depends only on the channel and noise characteristics, since $\mathbf{K}$ is a function of the channel and noise.

\section{PERFormance COMPARISON}

Fig. 4 depicts the magnitude response of the ANSI loop-3 channel, general MMSE equalizer, and subequalizer $C_{0}(k)$ proposed in this paper when the PCM-formatted signal of $f_{b}=8 \mathrm{kHz}$ is transmitted with $N=8, M=6$, and $L=256$, at an SNR of $45 \mathrm{~dB}$. Although the transmit signal has spectrum components of up to $4 \mathrm{kHz}$, each subequalizer does not need to compensate all the channel distortion, unlike a general MMSE equalizer that enhances the signal components attenuated by the channel. Since the $m$ th subequalizer needs to recover only the $m$ th nonzero symbols from every $N$ transmit symbol, it does not have to recover the signal component in the stopband. This process can easily be explained in the time domain. As can be seen in Fig. 5, the overall impulse response of the channel and subequalizer $c_{0}(t)$ has a value of one at index zero, and has zero magnitude elsewhere except when zeros are transmitted, and thus, the data $\left\{a_{0, k}\right\}$ can be transmitted without any interference.

The performance of the two equalizer schemes is compared in terms of the bit-error rate (BER) when applied to the PCM modem in several ANSI-loop models using a 64-PCM constellation mode. The 64-PCM constellation is selected from the code-level values of 46-78 and 175-206, having a minimum distance of 16 [2]. Note that, although the same signal constellation is applied to both equalizer schemes, the transmission rates are different, since the multiple-equalizer scheme sends only six symbols in every eight transmit symbol times. This results in a maximum transmission rate of $48 \mathrm{~kb} / \mathrm{s}$ for the V.92 equalizer, 


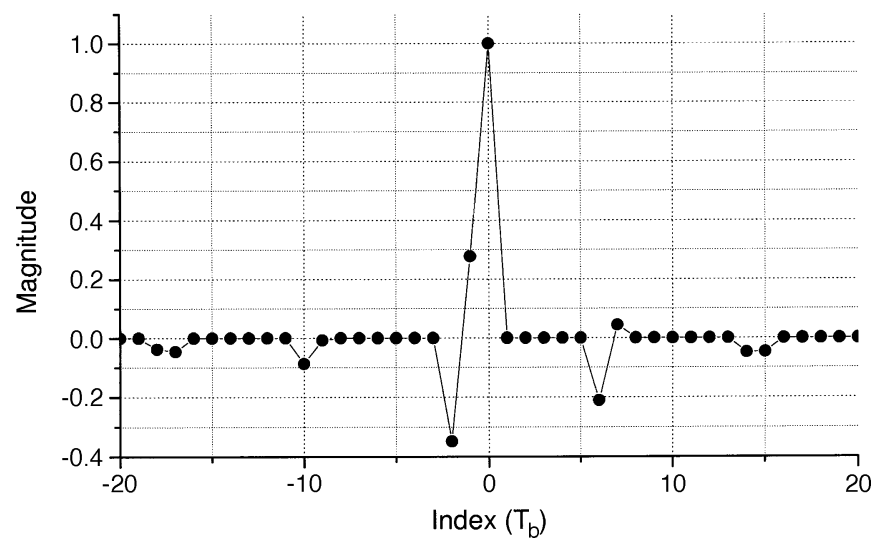

Fig. 5. Overall impulse response of loop 3 and subequalizer $c_{0}(t)$.

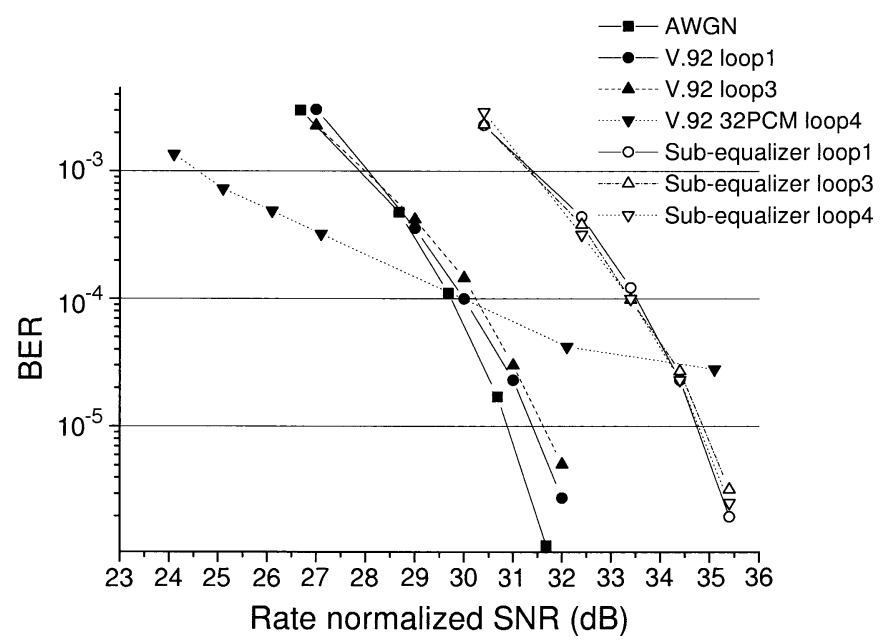

Fig. 6. BER performance of the proposed scheme.

and $36 \mathrm{~kb} / \mathrm{s}$ for the proposed subequalizer scheme. Thus, we compare the performance with respect to the rate-normalized SNR [14].

Fig. 6 depicts the BER performance in terms of the rate-normalized SNR. It can be seen that the V.92 equalizer performs better than the proposed subequalizer scheme by approximately $3 \mathrm{~dB}$ in loops 1 and 3 . This is due to the difference of the transmission rates of the two schemes. However, when the channel condition becomes poor, the performance of the V.92 equalizer deteriorates rapidly. As can be seen in Fig. 6, unlike the proposed subequalizer scheme, the V.92 equalizer even cannot receive the 64-PCM format signal, and it can barely receive the 32-PCM signal with poor performance in loop 4 . This performance degradation is mainly due to the tap length of the pre-equalizers not being long enough to compensate the channel distortion. As the number of taps for the IIR filter increases, it can be expected that the performance can be improved.

Fig. 7 examines the performance of the V.92 equalizer in loops 4 and 5. It can be seen that the V.92 equalizer can barely receive 32-PCM signal using a large tap size in loop 4, but it cannot in loop 5. Since the channel condition deteriorates as the loop number increases, it is expected that V.92 equalizer can not receive the signal in loops 5,6 , and 7 . This result indicates that the V.92 equalizer can not properly work on the loops 4-7, and thus, the V.92 modem should switch to the V.34 mode on these

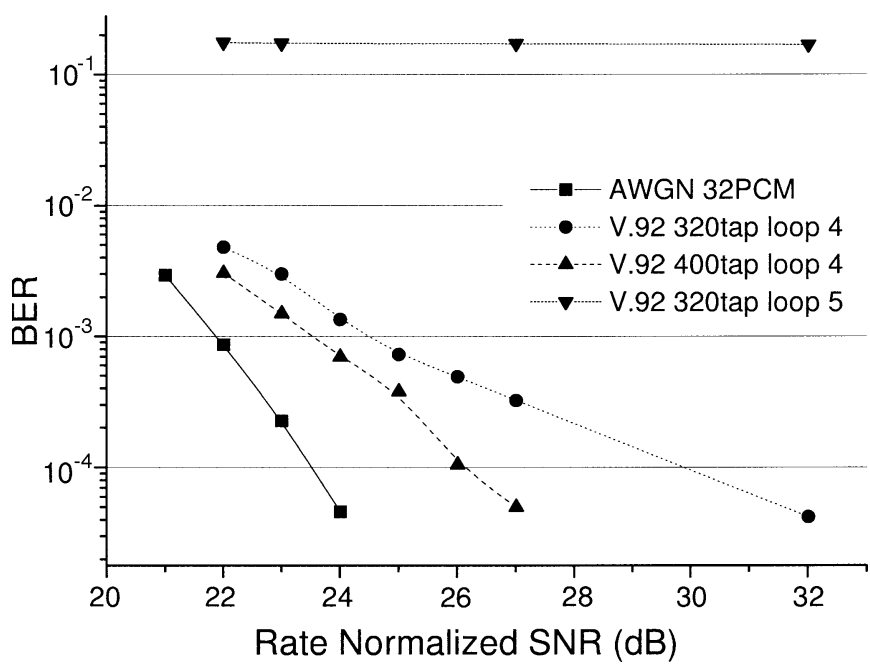

Fig. 7. BER performance of V.92 scheme for 32-PCM signal.

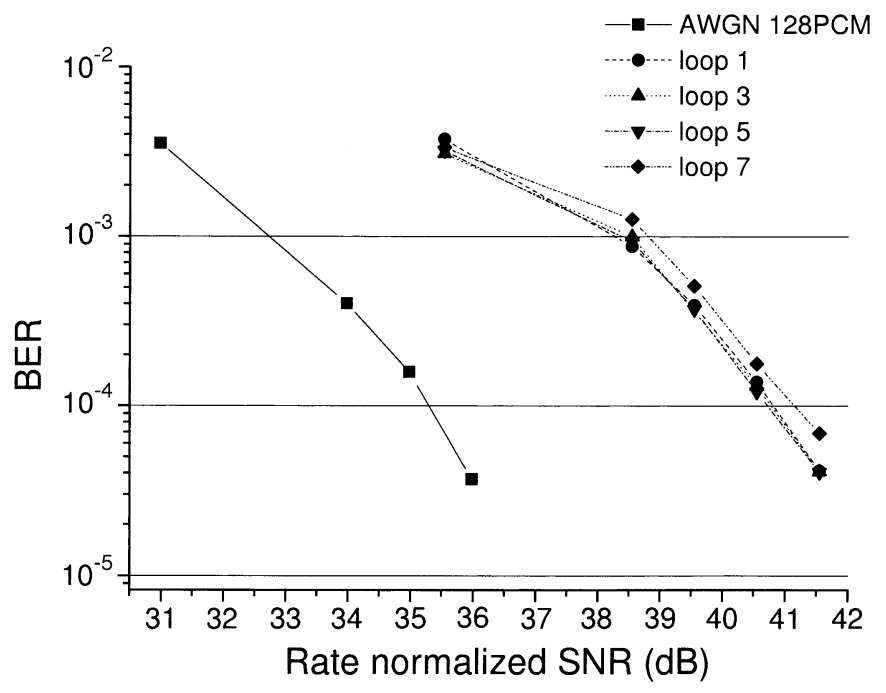

Fig. 8. BER performance of the proposed subequalizer scheme for 128-PCM signal.

TABLE I

COMPUTATIONAL LOAD FOR GENERATING A Single PCM SYMBOL

\begin{tabular}{c|c|c|c}
\hline & Required Memory & Multiplication & Addition \\
\hline V.92 & 1920 & 1280 & 1280 \\
\hline Proposed & 1536 & 96 & 96 \\
\hline
\end{tabular}

channels. Fig. 8 shows that, unlike the V.92 equalizer, the proposed subequalizer scheme is working well, even with the use of 128-PCM signal in ill-conditioned channels as in loops 5-7, which yields a transmission throughput of $42 \mathrm{~kb} / \mathrm{s}$.

As another measure, the computational complexity is compared for normal data transmission in the uplink. We assume the use of the two 320-tap IIR filters of the direct form II [15] in the V.92 scheme, and the use of the six 128-tap FIR-type subequalizers. As summarized in Table I, the proposed subequalizer scheme requires less memory and computational complexity than the V.92 pre-equalization scheme. Note that each subequalizer in the multiple subequalizer scheme needs to operate only once in every $N$-symbol time. This operation can be 
further reduced by noting that there are periodic zeros in the tap-delay line of the equalizer. The reduction of computational complexity makes the proposed subequalizer scheme quite attractive for DSP implementation.

\section{CONCLUSION}

In this paper, we have considered the use of multiple subequalizer scheme that can be applicable to the V.92 modem in lieu of the IIR-type equalizer scheme. The coefficient of the proposed subequalizer is optimally designed in the MMSE sense. Numerical results show that the proposed subequalizer scheme can provide performance quite robust to the channel condition, while the V.92 equalizer scheme works when the channel condition is mild. Moreover, the complexity of the proposed scheme is less than that of the V.92 equalizer scheme.

\section{APPENDIX}

DERIVATION OF (10)

By definition of $w_{m}(t)$ in (1), the sample of the equalized pulse at time $t=n T_{b}$ is

$$
w_{m}\left(n T_{b}\right)=\int h\left(n T_{b}-t\right) c(t) d t
$$

so that we can rewrite (6) as

$$
\begin{array}{r}
\sum_{n=-\infty}^{\infty} \sum_{\ell=0}^{M-1} w_{m}\left(n T-\ell T_{b}\right) h\left(n T-\ell T_{b}-\tau\right)+\sigma^{2} c_{m}(t) \\
=h\left(m T_{b}-t\right)
\end{array}
$$

The coefficient $c_{m}(t)$ of the $m$ th subequalizer is given by solving for $c_{m}(t)$ in (A2), as shown in (7). Since $\alpha_{m}(i)$ in (7) involves $f_{m}\left(i T_{b}\right)$ which is a function of $c_{m}(t)$ itself, $c_{m}(t)$ cannot be expressed in an explicit form. The MMSE equalizer $c_{m}(t)$, or equivalently, the coefficient $c_{m}(i)$ can be obtained as a function of the channel correlation and noise characteristics in the frequency domain.

Substituting $c_{m}(t)$ of (7) into (6) and with the definition of (12), we have

$$
\begin{gathered}
\int \sum_{i=-\infty}^{\infty}\left[\sum_{n=-\infty}^{\infty} \sum_{\ell=0}^{M-1} h\left(n T-\ell T_{b}-t\right) h\left(n T-\ell T_{b}-\tau\right)\right. \\
\left.\quad+\sigma^{2} \delta(t-\tau)\right] \alpha_{m}(i) s(i) h\left(i T_{b}-\tau\right) d \tau \\
=\sum_{i=-\infty}^{\infty}\left[\sum_{k=-\infty}^{\infty} \sum_{\ell=0}^{M-1} r_{h}(N k-i-\ell) h\left(N k T_{b}-\ell T_{b}-t\right)\right. \\
\left.\quad+\sigma^{2} h\left(i T_{b}-t\right)\right] \alpha_{m}(i) s(i) \\
=h\left(m T_{b}-t\right) .
\end{gathered}
$$

Taking the Fourier transform of (A3), we have

$$
\begin{gathered}
\sum_{k=-\infty}^{\infty} \sum_{i=-\infty}^{\infty} \sum_{\ell=0}^{M-1} r_{h}(N k-i-\ell) \alpha_{m}(i) s(i) e^{-j 2 \pi f\left(N k T_{b}-\ell T_{b}\right)} \\
+\sigma^{2} \sum_{i=-\infty}^{\infty} \alpha_{m}(i) s(i) e^{-j 2 \pi f i T_{b}}=e^{-j 2 \pi f m T_{b}}
\end{gathered}
$$

where we assume that $H(f) \neq 0$ for all $f$. The first term on the left-hand side of (A4) can be rewritten by splitting the exponential as

$$
\begin{aligned}
& \sum_{k=-\infty}^{\infty} \sum_{i=-\infty}^{\infty} \sum_{\ell=0}^{M-1} r_{h}(N k-i-\ell) \alpha_{m}(i) s(i) e^{-j 2 \pi f\left(N k T_{b}-\ell T_{b}\right)} \\
& =\sum_{\substack{k=-\infty \\
i=-\infty}}^{\infty} \sum_{\ell=0}^{M-1} r_{h}(N k-i-\ell) e^{-j 2 \pi f\left(N k T_{b}-\ell T_{b}-i T_{b}\right)} \\
& \quad \times \alpha_{m}(i) s(i) e^{-j 2 \pi f i T_{b}} .
\end{aligned}
$$

Expanding (A5) with respect to $\ell$, we have

$$
\begin{aligned}
\sum_{k=-\infty}^{\infty}\left[\sum_{i=-\infty}^{\infty} r_{h}(N k-i) e^{-j 2 \pi f\left(N k T_{b}-i T_{b}\right)}\right. \\
\quad \times \alpha_{m}(i) s(i) e^{-j 2 \pi f\left(N k T_{b}-\ell T_{b}\right)} \\
+\sum_{i=-\infty}^{\infty} r_{h}(N k-i-1) e^{-j 2 \pi f\left(N k T_{b}-i T_{b}-T_{b}\right)} \\
\quad \times \alpha_{m}(i) s(i) e^{-j 2 \pi f\left(N k T_{b}-\ell T_{b}\right)} \\
+\sum_{i=-\infty}^{\infty} r_{h}(N k-i-2) e^{-j 2 \pi f\left(N k T_{b}-i T_{b}-2 T_{b}\right)} \\
\quad \times \alpha_{m}(i) s(i) e^{-j 2 \pi f\left(N k T_{b}-\ell T_{b}\right)} \\
+\sum_{i=-\infty}^{\infty} r_{h}(N k-i-3) e^{-j 2 \pi f\left(N k T_{b}-i T_{b}-3 T_{b}\right)} \\
\times \alpha_{m}(i) s(i) e^{-j 2 \pi f\left(N k T_{b}-\ell T_{b}\right)} \\
\quad \vdots \\
+\sum_{i=-\infty}^{\infty} r_{h}(N k-i-(M-1)) \\
\times e^{-j 2 \pi f\left(N k T_{b}-i T_{b}-5 T_{b}\right)} \\
\times \alpha_{m}(i) s(i) e^{-j 2 \pi f\left(N k T_{b}-\ell T_{b}\right)}
\end{aligned}
$$

Noting that

$$
\begin{array}{r}
\sum_{k=-\infty}^{\infty} r_{h}(N k-i) e^{-j 2 \pi f\left(N k T_{b}-i T_{b}\right)}=\sum_{k=-\infty}^{\infty} r_{h}(N k-(N j+i)) \\
\times e^{-j 2 \pi f\left[N k T_{b}-(N j+i) T_{b}\right]}, \quad n=0,1, \ldots, N-1
\end{array}
$$

and that $s(i)$ has $(N-M)$ zeros in every $N$ positions, (A6) can be rewritten by collecting the terms with $r_{h}(N k-n)$ for $n=0,2, \ldots,(N-1)$ as

$$
\begin{aligned}
\sum_{k=-\infty}^{\infty}[ & r_{h}(N k-0) e^{-j 2 \pi f\left(N k T_{b}-n T_{b}\right)} \\
& \times \sum_{i=-\infty}^{\infty} \alpha_{m}(i) p_{0}(i) e^{-j 2 \pi f i T_{b}} \\
& +r_{h}(N k-1) e^{-j 2 \pi f\left(N k T_{b}-n T_{b}\right)} \\
& \times \sum_{i=-\infty}^{\infty} \alpha_{m}(i) p_{0}(i) e^{-j 2 \pi f i T_{b}} \\
& +r_{h}(N k-2) e^{-j 2 \pi f\left(N k T_{b}-n T_{b}\right)} \\
& \times \sum_{i=-\infty}^{\infty} \alpha_{m}(i) p_{0}(i) e^{-j 2 \pi f i T_{b}}
\end{aligned}
$$



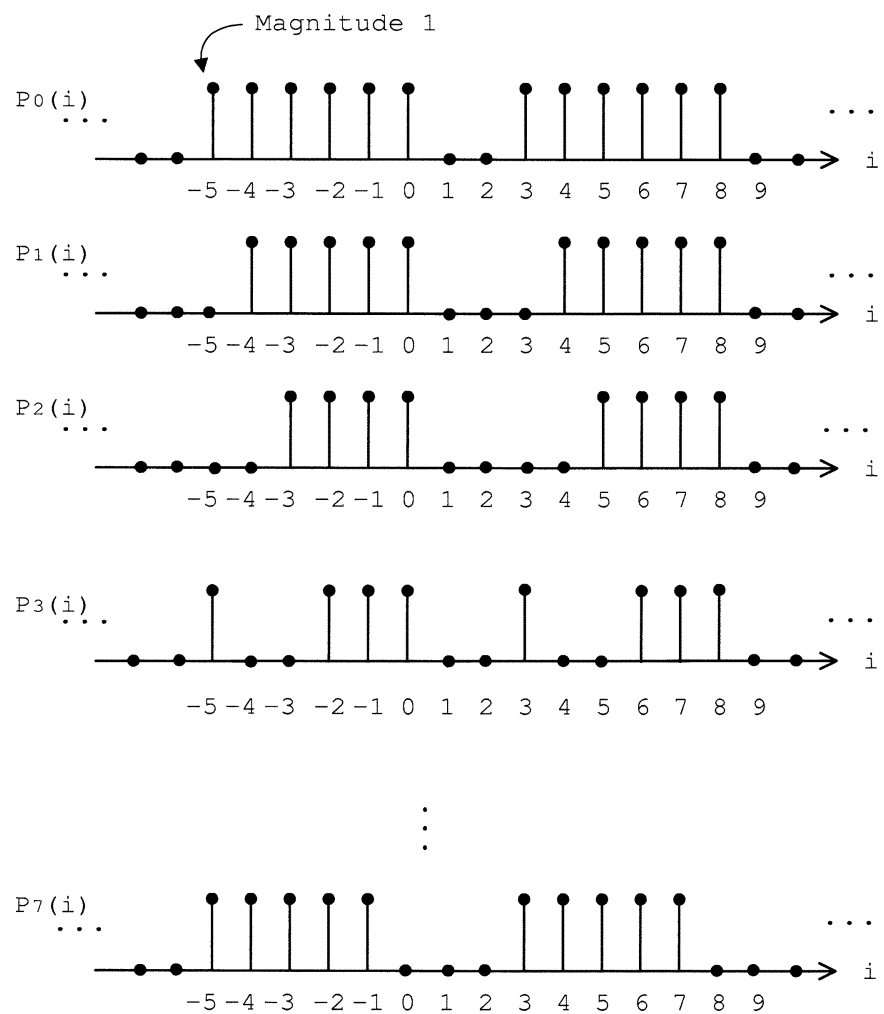

Fig. 9. Sequence $p_{n}(i)$ for $n=0,1,2, \ldots,(N-1)$ when $N=8$.

$$
\begin{aligned}
& +r_{h}(N k-(N-1)) e^{-j 2 \pi f\left(N k T_{b}-n T_{b}\right)} \\
& \left.\times \sum_{i=-\infty}^{\infty} \alpha_{m}(i) p_{0}(i) e^{-j 2 \pi f i T_{b}}\right] \\
& =\sum_{k=-\infty}^{\infty} \sum_{n=0}^{N-1}\left[r_{h}(N k-n) e^{-j 2 \pi f\left(N k T_{b}-n T_{b}\right)}\right. \\
& \left.\times \sum_{i=-\infty}^{\infty} \alpha_{m}(i) p_{n}(i) e^{-j 2 \pi f i T_{b}}\right]
\end{aligned}
$$

where $p_{n}(i)$ is a periodic sequence of ones defined as

$$
p_{n}(i) \equiv s(i) s(i-n), \quad n=0,1, \ldots, N-1 .
$$

As an example, Fig. 9 depicts $p_{n}(i)$ when $N=8$.
Using the fact that $p_{0}(i)=s(i) s(i-0)=s(i)$, and substituting (A7) into (A4), we have

$$
\begin{gathered}
\sum_{k=-\infty}^{\infty} \sum_{n=0}^{N-1}\left[r_{h}(N k-n) e^{-j 2 \pi f\left(N k T_{b}-n T_{b}\right)}\right. \\
\left.\times \sum_{i=-\infty}^{\infty} \alpha_{m}(i) p_{n}(i) e^{-j 2 \pi f i T_{b}}\right] \\
+\sigma^{2} \sum_{i=-\infty}^{\infty} \alpha_{m}(i) p_{0}(i) e^{-j 2 \pi f i T_{b}}=e^{-j 2 \pi f m T_{b}} .
\end{gathered}
$$

This can be rewritten as (10) with the definition of (11).

\section{REFERENCES}

[1] A Digital Modem and Analogue Modem Pair for Use on the Public Switched Telephone Network (PSTN) at Data Signaling Rates of Up to $56000 \mathrm{bit} / \mathrm{s}$ Downstream and Up to $33600 \mathrm{bit} / \mathrm{s}$ Upstream, ITU-T (CCITT) Recommendation V.90, Sept. 1998.

[2] R. L. Freeman, Telecommunication Transmission Handbook. New York: Wiley, 1976.

[3] Transmission Performance Characteristics of Pulse Code Modulation, ITU-T (CCITT) Recommendation G.712, Sept. 1992.

[4] Enhancements to Recommendation V.90, ITU-T (CCITT) Recommendation V.92, July 2001.

[5] A Modem Operating at Data Signaling Rates of Up to $33600 \mathrm{bit} / \mathrm{s}$ for Use on the General Switched Telephone Network and on Leased Point-to-Point 2-Wire Telephone-Type Circuit, ITU-T (CCITT) Recommendation V.34, Feb. 1998.

[6] E. Ayanoglu, N. R. Dagdeviren, G. D. Golden, and J. E. Mazzo, "An equalizer design technique for the PCM modem: A new modem for the digital public switched network," IEEE Trans. Commun., vol. 46, pp. 763-774, June 1998.

[7] I. Kalet, J. E. Mazo, and B. R. Saltzberg, "The capacity of PCM voiceband channels," in Proc. Int. Conf. Communications, July 1993, pp. 507-511.

[8] E. Ayanoglu, N. R. Dagdeviren, J. E. Mazzo, and B. R. Saltzberg, "A high-speed modem synchronized to a remote CODEC," U.S. Patent 5,394,437, Feb. 28, 1995.

[9] H. Nyquist, "Certain topics in telegraph transmission theory," Trans. AIEE, vol. 47, pp. 617-644, Feb. 1928.

[10] J. L. Yen, "On the nonuniform sampling of bandwidth-limited signals," IRE Trans. Circuit Theory, vol. CT-3, pp. 251-257, Dec. 1956.

[11] E. Ayanoglu, "Data transmission when the sampling frequency exceeds the Nyquist rate," IEEE Commun. Lett., vol. 1, pp. 157-159, Nov. 1997.

[12] V. Eyuboglu and J. D. Forney, "Trellis precoding: Combined coding, precoding and shaping for intersymbol interference channels," IEEE Trans. Inform. Theory, vol. 38, pp. 301-314, Mar. 1992.

[13] R. D. Gitlin, J. F. Hayes, and S. B. Weinstein, Data Communications Principles. New York: Plenum, 1992.

[14] J. G. Proakis, Digital Communications, 2nd ed. New York: McGrawHill, 1989.

[15] A. V. Oppenheim and R. W. Schafer, Discrete-Time Signal Processing. Englewood Cliffs, NJ: Prentice-Hall, 1989. 